It has been common throughout history to ascribe the worst possible characteristics to our enemies and then use those enemies as exemplars of erroneous behavior. The British have, from time to time, made enemies of almost everyone in the world, so their language (i.e., English, possibly as distinct from "American") is particularly rich with examples, often directed at the one most consistent enemy from medieval times until the dawn of the 20th century: the French. Unauthorized absences are known as "French leave," for example. For their part, the French are wont to disparage English cooking by referring to the water that vegetables are boiled in as sauce Anglaise.

The United States is big enough to sustain several internal examples of this kind of thing, most frequently related to that bastion of American culture, the automobile. A "Kentucky right turn" involves a swing to the left of the road followed by a turn to the right, while a "California stop" involves rolling through a stop sign without ever achieving zero velocity. My own observation is that these behaviors are no more prevalent in their supposed home states than in the rest of the country.

\section{Fighting Words Or How to Spot the Real Enemy}

A more subtle form of rivalry plays out in the language heard within the vaulted halls of science and engineering. Lack of "chemistry" is often cited as a reason for the breakdown of a group dynamic, but in this example, "chemistry" is just as often used in a positive sense, so maybe this is not a case of non-chemists shifting blame to chemistry. On the other hand, I recently heard a researcher, engaged in the cleaning out of a laboratory sink, complain that "things are getting a bit biological in here," and, as you may guess, he was a physical scientist. A meeting of engineering academics provided an interesting insight, after a decision-making process went astray: A mechanical engineer declared that the process had been "short-circuited" while an electrical engineer complained that it had "slipped a cog."

We might expect materials science and engineering, in all of its wonderful interdisciplinarity, to provide many examples of this subtle form of blame-shifting, but in fact it is very hard to find examples of a metallurgist using a ceramic metaphor in describing a problem, or a polymer sci- entist shifting the blame to semiconductors. Just think, a manager exhibiting too much rigidity might be "a bit too $s p^{3}$ bonded" or perhaps "too highly crosslinked." We all tend, instead, to go after nature itself. When something fails for lack of enough people on the team, we refer to a "critical mass" problem. When things descend into chaos, we are apt to describe the situation problem in terms of entropy. When, despite our best efforts, something cannot be made to work at all, we may blame the energy balance.

So, to leap to a thoroughly unjustified conclusion, we have met the enemy, and (for once) it is not us. Our rhetoric seems to imply that our efforts revolve around a common cause of wresting the best materials properties from nature, despite its best efforts to thwart us, and we focus our enmity on nature rather than upon each other. More than the creation of any unified academic department, or textbook, or any distinguished proclamation, this aspect of our behavior clearly indicates that materials science is a unified culture without serious internecine rivalries.

Alex KING 